This paper is given as a first draft of a framework better clarified in the following contributions

\author{
The potential of a thick present \\ through undefined causality and non-locality
}

Entropy 2022, 24(3), 410 - doi.org/10.3390/e24030410

and

The Universe as a Telecommunication Network pre-print accepted at DICE2022 conference - doi.org/10.31219/osf.io/c7bg4 


\title{
A simple holographic universe
}

\author{
Alessandro Capurso \\ Mar. 15, 2022 - Rome, Italy
}

\begin{abstract}
In the context of an evolution occurring in thick present instants, we investigate the possible emergence of a spacelike foliation through a toy model based on the information of entanglement among virtual quanta of space. We elaborate on the hypotheses looking for physically implementable solutions and identify the need for finite and discrete information, relativity of the information in respect to global scales of reference and logical consistency of the global information. We conjecture the emergence of an imaginary spacetime as a holographic boundary between two symmetric bulk regions extending in the thick present and evolving synched on an atomic cycle (intended as a spacetime "sampling-rate" and identifying an elementary quantum of action). Within the thick present, we propose how the information of a massive particle could be described through an analysis of scale of the information of non-locality (entanglement in the space foliation). Finally, we conjecture how this information could be intended and efficiently encoded in the symmetric bulks of the present through a wavelet analysis (logarithmic perspective) and elaborate on the possible features emerging from the symmetries in the model (quantum spin and matter/anti-matter).
\end{abstract}

Keywords: thick present; information; entanglement; CTC; spacetime; matter; toy model.

INTRODUCTION

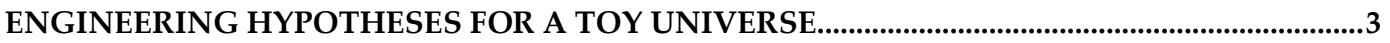

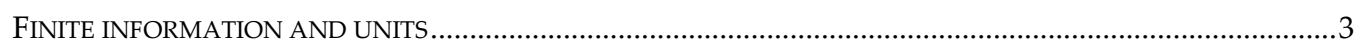

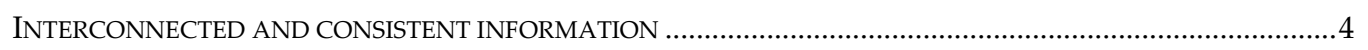

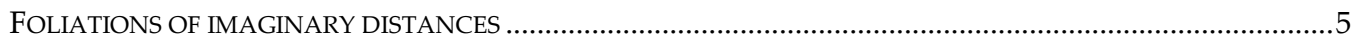

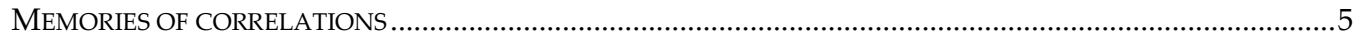

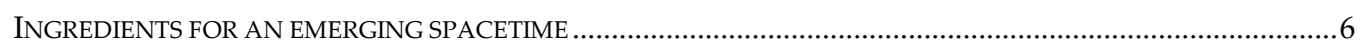

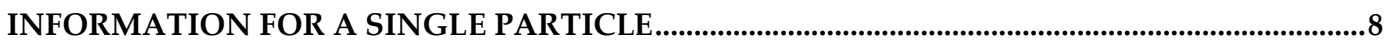

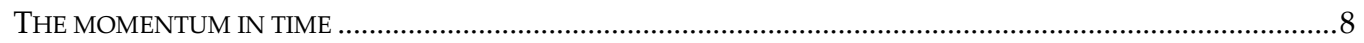

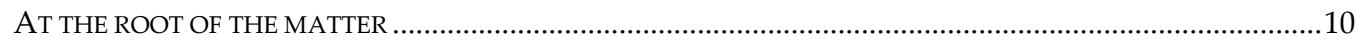

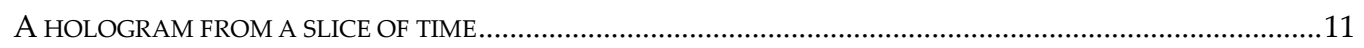

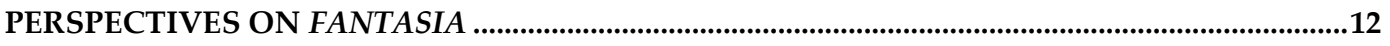

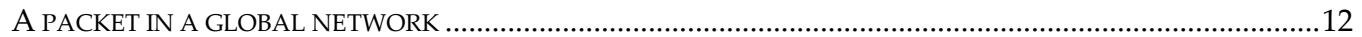

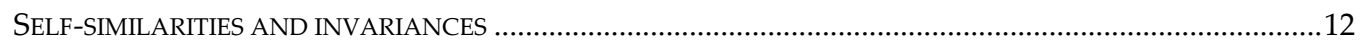

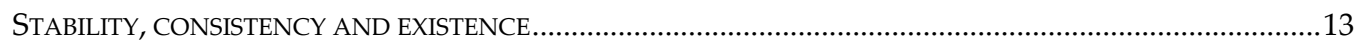

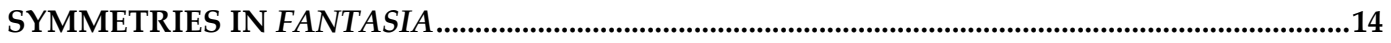

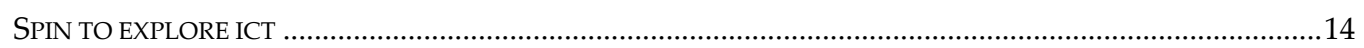

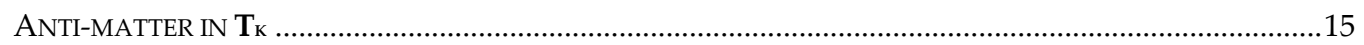

SYNTHESIS AND OUTLOOK ....................................................................................................................16

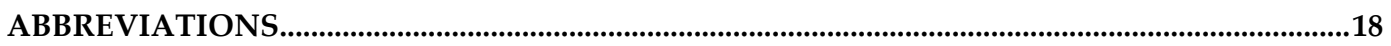

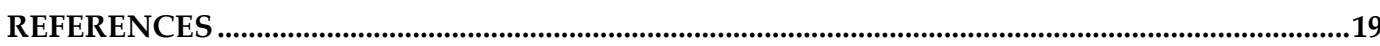

\# ale.capurso@gmail.com - orcid.org/0000-0003-3277-3588 


\section{Introduction}

Many research paths towards a theory of Quantum Gravity (QG), able to describe spacetime and the Universe evolution in a quantum formalism and give a more general framework to the current theories, start from the concept of information as a lens through which both quantum mechanics $(\mathrm{QM})$ and spacetime should be interpreted.

It is worth investigating the description of a toy universe in which spacetime, instead of being just a static background for QM, could emerge from information. Starting with a toy model inspired by Information and Communication Technologies (ICT) and Quantum Information Science (QIS), and understanding which hypotheses could be needed for an implementable solution, might give insights on how our Universe, when described through information, could behave.

This contribution follows a previous work, reported in Ref. [1], in which we have introduced a possible interpretation on the nature of time and entanglement. In the context of a spacetime described through information, we have identified in a thick present the only element of reality in time. The concept of superposed imaginary paths developing in a spacelike foliation and closing in the thickness of the present in Closed Time-like Curves (CTC) allowed us to consider entanglement (in time order or spatial position) as the logically consistent information potential of an "open choice", related to undefined causality and non-locality in each instant.

In the first part of this new work, we will reason on the hypotheses needed to describe, from the perspective of a physically implementable system, a discrete and finite spacelike foliation emerging from an information potential in the thick present (intended as entanglement among spatially distant quanta of space).

We will then elaborate on how a massive particle could be represented in the context of this thick present potential, defining a relation between a "proper tempo" of the particle and the information of non-locality in the wave function. To better understand the insights offered by the model, we will consider multiple perspectives on how this information should be intended, proposing also how it could be efficiently encoded.

Finally, we will investigate the emerging features intrinsic in the symmetries of the model.

From now on, we will refer to the proposed model as Fantasia, a tribute to the universe where every story could exist. The reader should be warned that the concept of a "spacetime from information" and the reasoning on the possible encodings of it are not promoting the idea that "we live in a simulation", which is an unneeded speculation.

\section{Engineering hypotheses for a toy universe}

\section{Finite information and global units}

In the context of "spacetime from information", to have a physically realizable universe, we need to upper bound the information required to describe it. Actually, no implementable or existing universes should need infinite or unbounded information to be physically representable.

From a mathematical perspective, a finite information cannot describe the Real continuum (as also debated in [2]), hence the spacetime fabric in Fantasia is considered discrete, and its physical observables must be relative to global units of reference.

In a discrete spacetime, it seems logic to consider the passage of time as occurring in discrete steps, below which it is meaningless to define the concept of time. Moreover, in the context of a universe based on information, what should matter is the current perspective on information and no precise reconstruction of spacetime at a given far past instant should be required. Information of the far past states is not needed beyond the current state dependencies (as a causal relation of the current state with the far past ones) and what happened in the past states cannot be changed. Only a summary of the causal past dependencies is needed, further limiting the growth of required information. On the same line, we can consider that no information about the future should be needed for a consistent emergence of spacetime, as the future is intended as still undefined and open. 
The hypothesis of a discrete passage of time in the context of a fixed past and an open future has already been introduced in Ref. [1] (following [2], [3], [4], [5], [6], [7] and [8]), where we have concluded the existence of a thick present as the only element of reality in an emerging axis of time. A thick present is intended as a time symmetric information potential, existing between $-T$ and $+T$, from which a spacelike foliation emerges at $2 k T$. In a QIS perspective, a thick present has been considered as a discrete elaboration cycle of the global information potential. Its temporal extension spans from $(2 k-1) T$ to $(2 k+1) T$ and is interpreted as a global reference for time intervals (not in contrast with a relativistic description of time) as well as a spacetime information "sampling rate".

The present evolution cycle has been considered as a delta of action at Planck scale, which acts as an absolute reference for the information potential (as a global "common beat").

This reference delta of action could allow a coherent decoding of the whole spacetime and, from a local perspective, the coherent decoding of the surroundings. We wil consider the reduced Planck constant $\hbar$ as the simplest reference needed to properly define a relative confrontation in terms of unitary action. It is an absolute reference related to the Planck units of mass, spatial distance, and time, and could be interpreted as a "common beat", characteristic of the universe and consistent in every point in the emerging spacetime.

Towards a discrete passage of time and a discrete space, also mass should be considered from a perspective relative to some common reference. The relevance of the Planck mass in the evaluation of the discrete nature of time has also been recently highlighted in Ref. [9] and [10].

We could consider the Planck units as universal references (in Fantasia as in our Universe). Giving Planck length $L$, Planck mass $M$, Planck time $T$ and considering $F=1 / T$ as the ideal maximum freedom in $f$ (angular frequency and related rate of change, frequency for short), we can express the fundamental constants (from which they are derived) as following:

$$
c=\frac{L}{T}=L F ; \quad \hbar=\frac{M L^{2}}{T}=M L^{2} F=M L c ; \quad G=\frac{L}{M} c^{2}
$$

As a final thought, the conceptual discovery of the Planck units in our Universe seems a strong indication towards both a discreteness of spacetime and the existence of a possible absolute perspective, with a minimum "reference rod" for spatial distance and a global "tick", "beat" or minimum "blink between observations" for time intervals.

Eventually, only with absolute references it is possible to describe meaningfully, coherently and consistently any difference among relations or relativistic perspectives on the surrounding spacetime from 2 independent observers.

\section{Interconnected and consistent information}

The concept of "observer" is key in a QM description of phenomena, but its meaning has always been open to several interpretation. In a context of "it from bit", questioning if the underlying information and the emerging spacetime are independent from the observers should be meaningless: there are no external observers or isolated labs in a universe without a "spacetime stage", as all observers are part of the information from which the observed phenomena emerge. Observers could make local measurements, relative to their point of view on spacetime, but the concept of relativity itself seems to require the emerging spacetime to be interconnected at a more fundamental level. Actually, to be relative, any kind of information cannot be isolated or external, at least in respect to a global perspective, as it is in the confrontation with a global perspective that all single local ones find their relative coherence and the global one finds its consistency. Eventually, it could be in the limited perspective of "isolated systems" that many theories on spacetime find hard to "go quantum", and a local and a global consistency might be lost.

In Ref. [1], we have studied the relation among entanglement, non-locality, undefined causality, and logical consistency. Starting from Ref. [11], [12], [13], [14], [15], [16], [17], [18] and [19], we have considered entanglement as the information of the superposition of the outcomes of an "open choice", persisting in each present instant as a logically consistent potential that discards under/over-determined solutions. 
We have conjectured a relation between the information of entanglement and CTC, constructed as the superposition of a forward (causal) and a backward evolving wave in a time-symmetric thick present, representing undefined causality and non-locality.

These time-symmetric waves from $-T$ and $+T$ closed in the present as CTC are considered equivalent to "memory loops", able to encode in each instant the logically consistent information of an "open choice" (spatial position or temporal order) of which the outcome is undetermined at the most fundamental level.

Given these considerations, the information (as well as the observables) in Fantasia must be logically consistent, with no contradiction between a local and relative point of view and a global one. Logical consistency requires that information shall not be under or over determined and it is a weaker requirement than causality, as debated in Ref. [11].

The use of absolute references to describe observables and of CTC as memory loops to model entanglement should assure the logical consistency of the global information considered.

\section{Foliations of imaginary distances}

In a discrete evolution, we could consider the concept of spatial distance within a given cycle deriving its physical meaning thanks to an absolute speed of light: we could define distance as "the imaginary time needed for light to cover that distance". If the speed of light were not an absolute reference for observers, there would be no coherent spatial distances and no perception of a coherent relativistic and causal spacetime.

From the idea of an imaginary time of motion and the imaginary distance ir between 2 points at the speed of light, we can construct an imaginary space foliation at a given instant considering, from any possible point as origin, the space described through ir and 2 angular degrees of freedom. Note that, in a discrete axis of spatial distances in a thick present of $2 \mathrm{~T}$, a point should be intended as a quantum of space, with a minim radial extension of $L$.

The concept of an emerging reality connected to imaginary paths was introduced in QM as a Path Integral (PI) formulation by Feynman. The description of a spacelike foliation through an imaginary time of motion has also been introduced in Ref. [1]. We will consider the imaginary coherent time $i c t=i r=i n L=i L e^{\varphi}$ as the axis of a discrete spatial distance variable (with $c$ equal to the speed of causality and light, $L$ the Planck length and being $i$ the imaginary unit). In a flat space defined through an imaginary coherent time ict, the conservation of causality is accounted for coherence in the contribution $(i c t)^{2}$ considering the causal propagation of the information along the "ticking" of present instants.

If there is entanglement among 2 spacelike separated regions, then distance should not only be considered through the propagation in a flat ict, but also through the connection in the thickness of the present instant. In a context of entanglement, non-locality and global logical consistency, it is as if a flat ict spatial axis had a limited perspective, giving the existing correlation encoded in the thickness of spacetime (as in Ref. [1]).

Note that non-local correlations between distant points are always related to interactions occurred in the past evolution cycles and cannot in any way allow faster than light communication. Having required logical consistency of the global information potential, causality violations are actually excluded a priori.

\section{Memories of correlations}

We have considered a universe emerging as a spacelike foliation from an information potential evolving in the thick present. We have elaborated on the possible requirements from a perspective focused on implementable systems (considering the use of discrete absolute units of reference) and required logical consistency of the information potential (for a coherent global perspective on spacetime). Still, we have to properly elaborate on the information we would need to generate the emerging spacetime of Fantasia.

As in several holographic approaches, we could consider the general idea of "connection among spacetime points" (intended as a link of correlation and entanglement among spatially distant quanta of space in a global network) as the elementary information that shapes and curves spacetime beyond an empty and flat imaginary space. 
From an ICT and QIS perspective, a simple model of correlation could rely on the sharing of a common phase or code in respect to a common external reference. In the simplest scenario, the external reference could be unique for all the spacetime points, becoming a "universal constant" in Fantasia. We will identify in the elementary quantum of action $\hbar$ the reference action given by the present information elaboration cycle. It is proposed as a "common beat" in Fantasia, acting as a global reference to weigh the information of correlations in spacetime, as for the imaginary action in the PI formulation.

We could then picture the imaginary space in Fantasia as a lattice of quanta evolving synched on the present instants and an interconnected information on a "common beat". In each imaginary quantum of space, the information of correlation with other quanta is scaled on the defined global reference of action, as better clarified in the coming chapters.

We will call "Absolute Time" (or $\boldsymbol{T}_{k}$ for short) a symmetric and "cyclic memory" axis (orthogonal to the ict axis of spatial distance) that encodes the information potential and from which, at each $2 k T$, the space foliation emerges. The word "absolute" shall not be intended in Newtonian terms but related to natural references (as the absolute zero) and to a perspective on the information potential scaled on absolute (Planck) units. The term "cyclic memory" is referred to the encoding of the information of correlation in space as "encoded in a quantum memory" between $(2 k-1) T$ and $(2 k+1) T$ in each $k^{\text {th }}$ elaboration cycle.

The description of spacetime and of the gravitational vacuum introduced so far resembles the "sea of virtual Planck-mass black holes" proposed by Hawking in Ref. [20]. The interpretation suggested here is to consider this "virtual sea" not only as static mass or spatial references, but as references for the evolution of information as well, connected to the absolute reference of action available in every quanta of space. In Fantasia, spacetime emerges as imaginary from the potential of the present $\boldsymbol{T}_{k}$ like a "sea of globally synched virtual Planck-mass oscillators" (as virtual clocks synched on a common beat) encoding the local perspective on the imaginary space ict in terms of entanglement with other imaginary quanta of space. When the imaginary paths among the quanta of space close in the thickness of the present, they connect these distant virtual Planck black holes with ER bridges of non-local potential, represented by CTC or "memory loops", as introduced in Ref. [1].

In a holographic description, common to several approaches to QG that consider spacetime as emerging from the entanglement among distant quanta of space, $T_{k}$ shall be intended as the "bulk", while the imaginary space foliation as the boundary emerging from the information encoded in the bulk. Given the time symmetric description in the thick present, the imaginary space emerging at $2 k T$ is actually at the boundary between 2 symmetric bulk regions, with the perspective from $(2 k-1) T$ to $2 k T$ and from $(2 k+1) T$ to $2 k T$. In every thick present instant, the Absolute Time memory axis $\boldsymbol{T}_{k}$ encodes and projects the information of entanglement in the foliation and repeats its cycles, allowing the persistence of the potential, its transformation in a probabilistic imaginary space and the evaluation of events as irreversible differences at the boundary of each present instant.

\section{Ingredients for an emerging spacetime}

After having discussed several aspects related to a possible spacetime emerging from information, it is worth summarizing the key conceptual assumptions considered in the proposed toy model. These concepts are also graphically illustrated in Figures 1 and 2.

- Spacetime in Fantasia depends on the information potential of entanglement among imaginary quanta of space. For a physically implementable solution, this information is considered to be finite, discrete, logically consistent and relative to global scales of reference (such as the Planck units $T, L$ and $M$ )

- Time, as an extended axis in its thermodynamic nature, is considered emergent. Only the current instant is given as ontologically existing. Evolution occurs in discrete steps and each instant is pictured as a thick present, lasting from (2k-1)T to $(2 k+1) T$ and connected to a spacetime information "sampling rate". Classical information is considered as an irreversible causal past (events of decoherence) 
- The entanglement information is considered encoded in closed paths (through a PI approach in a time symmetric description within the thickness of the present). These CTC express undefined causality and non-locality in the emerging imaginary space and assure logical consistency of the information potential. CTC are considered equivalent to "memory loops" in which the information potential of entanglement as an "open choice" persists in each thick present cycle

- The evolution of the information potential in each present instant (as the spacetime "processing cycle") identifies a universal reference of action available in ever point in the emerging space to scale the information potential of entanglement among the imaginary quanta. The emerging spacetime could be pictured as a "sea of virtual Planck black holes" synched on a "common beat" in an interconnected universe

- Flat space emerges every $2 k T$ as an imaginary spacelike foliation. It develops from all quanta of space as imaginary paths along an imaginary time of motion at $c$, in a resulting imaginary axis of spatial distance $i c t=i c n T$ and Minkowski metric

- Space is considered as a holographic boundary between a pair of symmetric bulk regions called Absolute Time or $T_{k}$. The non-local information of entanglement that curves the flat imaginary space ict is encoded in the symmetric bulks $T_{k}$ through CTC (equivalent to ER bridges between the distant virtual black hole quanta of space). The Absolute Time bulks from $(2 k-1) T$ and $(2 k+1) T$ are also intended as a "cyclic memory" (quantum buffer) of the information potential of entanglement

- There is no absolute perspective on the global information potential of the thick present encoded in $\boldsymbol{T}_{k}$ from any point within the imaginary space emerging at $2 k T$

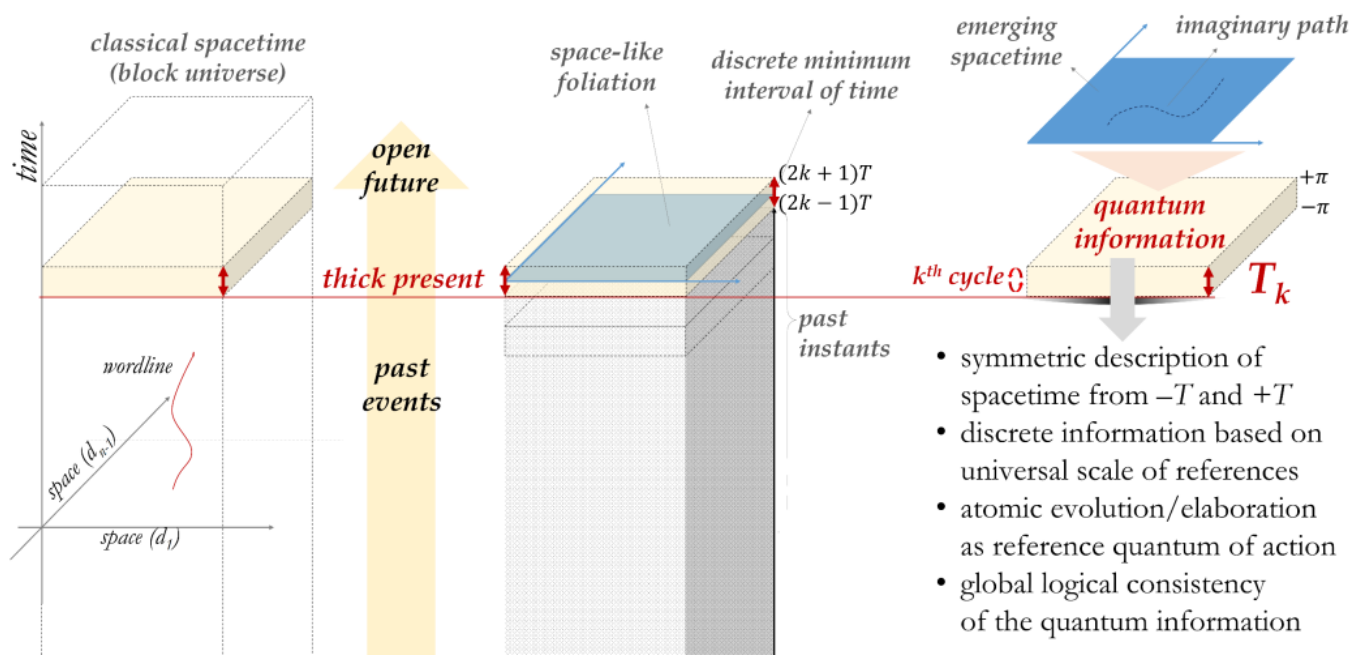

Figure 1. Identification of the thick present as the current thick spacelike foliation and corresponding $k^{\text {th }}$ elaboration cycle of the quantum information $\boldsymbol{T}_{k}$ from which spacetime is considered emerging.

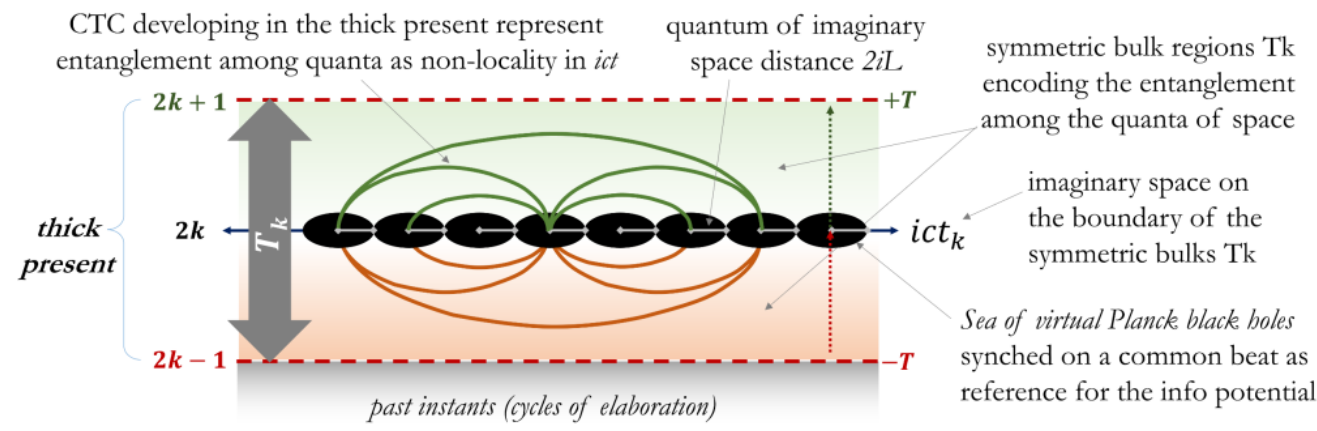

Figure 2. Imaginary space ict as a boundary between 2 symmetric bulks in the present. A reference of action is available in every quanta of space as in a sea of virtual Planck black holes synched on a common beat. CTC are ER bridges between the Planck black holes and represent entanglement in ict. 
We have proposed some high level "engineering hypotheses" for a toy model of a universe based on information, but we are still missing a proper description of the matter that curves the geometry of the emerging space and its connection with the info of entanglement among the imaginary quanta of space encoded in the symmetric bulks of $\boldsymbol{T}_{\boldsymbol{k}}$. In Ref. [1], we have proposed a possible relation among the information of non-locality encoded in the wave function and the entanglement in the imaginary space, conjecturing a possible extension of the equation derived in holographic theories to $E R=E P R=C T C @ \boldsymbol{T}_{k}$. Following this idea, we have suggested a description of the information of particles through a probabilistic bundle of CTC developing in the thick present, as in Figure 3.

In the next chapter, we will elaborate more on this idea and investigate how the information potential of a single particle could be described in the thick present of Fantasia. We will first consider the Einstein 4-vector through the momenta in $\boldsymbol{T}_{\boldsymbol{k}}$ and $i c t$, and then relate the wave function of an elementary particle to a vibrating mode in the Absolute Time in respect to the global reference of action.

The vibrating mode, as a particle "characteristic tempo" in the emerging spacetime, will be connected to the bundle of CTC entangling the space foliation, towards a description of particles as a "volume of space on a common beat" in respect to the global beat of reference in the thick present.

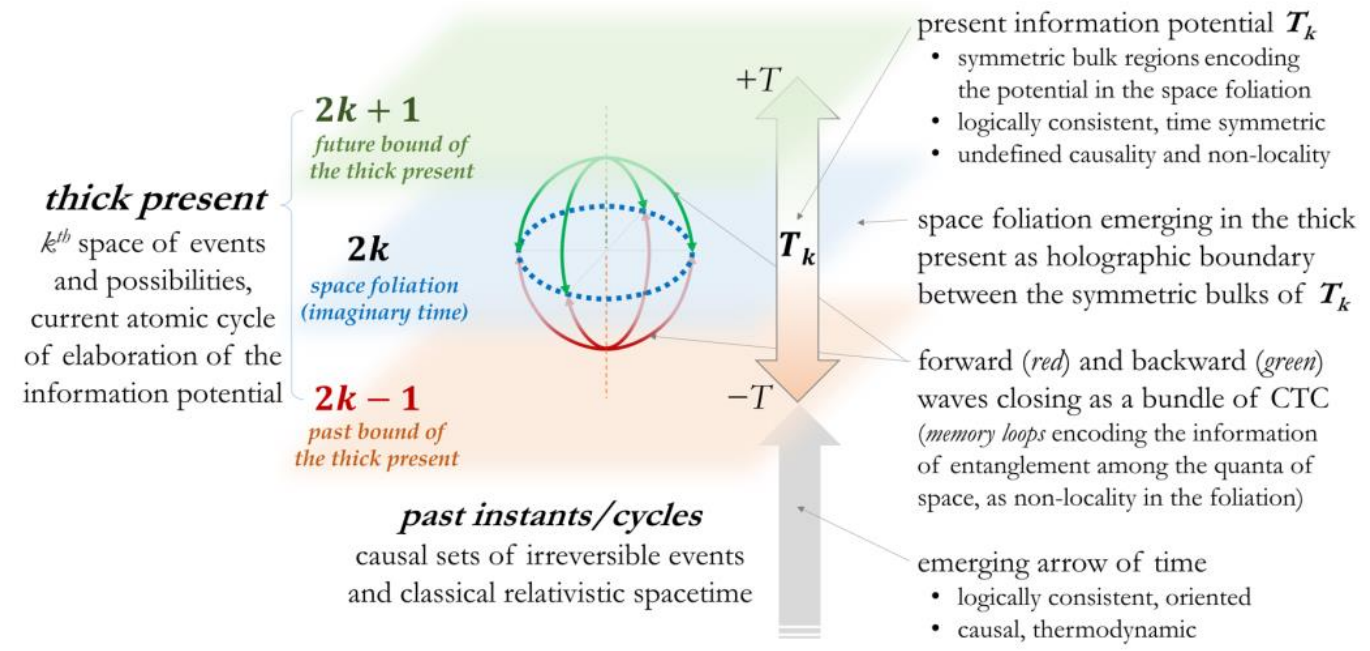

Figure 3. Graphics illustrating the space foliation at $2 k T$ in the thick present as the boundary between 2 symmetric bulk regions extending from $(2 k-1) T$ to $2 k T$ and from $(2 k+1) T$ to $2 k T$. The bulks encode the information $T_{k}$ (intended in a holographic perspective as entanglement among quanta of space) from which spacetime emerges. CTC in the thickness of the present represent non-local potential, while are equivalent to undefined orders of traversed points when developing in the imaginary time.

\section{Information for a single particle}

The momentum in time

In the present elaboration cycle, given a particle of relativistic mass $m$ that propagates in empty imaginary space with translational velocity $v_{s}$, consider the following equations:

$$
\beta=\frac{v_{S}}{c}=\sin \left(\tau_{s}\right) ; \quad \frac{1}{\gamma}=\sqrt{1-\beta^{2}}=\cos \left(\tau_{s}\right) ; \quad b=\beta \gamma=\tan \left(\tau_{s}\right)
$$

Consider then a momentum in time connected to the particle internal information. In a QM interpretation of the information potential, we could picture this momentum as an "internal pulse" connected to the often called zitterbewegung (trembling motion) observed from the Center of Mass (CoM) reference frame, different from $v_{s}$ that represents the translational velocity of the CoM in respect to an external frame of reference. 
Given $E$ as the total energy of the particle, we can explicitly define then two momenta:

$$
\begin{gathered}
p_{T}=\frac{E}{c} \cos \left(\tau_{s}\right)=\text { momentum in Time } \\
p_{S}=\frac{E}{c} \sin \left(\tau_{S}\right)=\text { momentum in Space } \\
E^{2}=\left(p_{T} c\right)^{2}+\left(p_{S} c\right)^{2}=\left(p_{S T}{ }^{*} c\right)\left(p_{S T} c\right)=\left|p_{S T} c\right|^{2}
\end{gathered}
$$

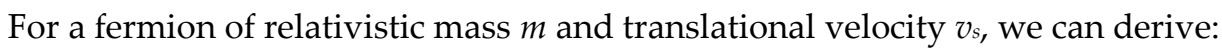

$$
\begin{gathered}
p_{T}=\frac{E}{c} \cos \left(\tau_{s}\right)=m c \cos \left(\tau_{s}\right)=m_{0} c \\
p_{S}=\frac{E}{c} \sin \left(\tau_{s}\right)=m \beta c=m v_{S}=m_{0} \gamma \beta c=m_{0} b c=b p_{T}=p_{T} \tan \left(\tau_{s}\right)
\end{gathered}
$$

For a photon, $\tau_{s} \rightarrow \pi / 2$ and, giving its frequency $f$, the considered momenta become:

$$
\begin{gathered}
p_{T}=\frac{E}{c} \cos \left(\tau_{s}\right)=\frac{\hbar f}{c} \cos \left(\tau_{s}\right)=\left(M \frac{f}{F}\right) c \cos \left(\tau_{s}\right) \rightarrow 0 \\
p_{S}=\frac{E}{c} \sin \left(\tau_{s}\right)=\frac{\hbar f}{c}=\left(M \frac{f}{F}\right) c \sin \left(\tau_{s}\right) \rightarrow\left(M \frac{f}{F}\right) c
\end{gathered}
$$

If we consider the momentum in space as developing in a 3D imaginary space defined by 3 orthogonal imaginary components, the momentum in time develops then on the real axis, orthogonal to all 3 imaginary coordinates of space (as in quaternions). In a simpler and mono-dimensional space, we can see the momentum in space on the imaginary axis of spatial distance ict and the momentum in time on the orthogonal real axis, connected to the characteristic information of the particle persisting in $\boldsymbol{T}_{k}$.

Beyond a relativistic description, extending to $\mathrm{QM}$, consider $\psi_{E}=R e^{\frac{i S}{\hbar}}$ as the wave function describing the position of a massive particle. We can define a velocity $v_{q}$ connected to the internal degree of freedom within space and the spin of the particle (as proposed in Ref. [21] and [22]). We can consider $v_{q}$ as related to the momentum in time defined before, seen as the particle "beat in spacetime". For a fermion, given Equation 5, we can consider this "beat" equal to the speed of light in absolute terms (not intended as a particle travelling at the speed of light but as a "sampling frequency" of the zitterbewegung information potential in spacetime during each present instant).

$$
\left|v_{q}\right|=\hbar\left|\frac{\nabla R}{m_{0} R}\right|=c\left|\frac{M L}{m_{0}} \frac{\nabla R}{R}\right|=c\left|\frac{M L}{m_{0}} \nabla \ln R\right|=c
$$

We can then describe the momentum in time through the log-gradient of the amplitude of the wave function, while the momentum in space as the gradient of the phase.

$$
\begin{gathered}
p_{T}=\frac{E}{c} \cos \left(\tau_{s}\right)=m_{o} c=M L c\left(-\frac{\nabla R}{R}\right)=p_{0} L(-\nabla \ln R)=-\hbar \nabla \ln (R) \\
p_{S}=\frac{E}{c} \sin \left(\tau_{s}\right)=m v_{S}=\nabla S
\end{gathered}
$$

Elaborating on the last equations, we can better express the relation between the particle's wave function and the momenta introduced.

$$
\begin{gathered}
\nabla \psi_{E}=\nabla e^{\left(\ln (R)+\frac{i S}{\hbar}\right)}=\left(\nabla\left(\ln (R)+i \frac{S}{\hbar}\right)\right) R e^{\frac{i S}{\hbar}}=-\frac{\left(p_{T}-i p_{S}\right)}{\hbar} \psi_{E}=-\frac{p_{S T}{ }^{*}}{\hbar} \psi_{E} \\
\left(-p_{S T}{ }^{*}\right) \psi_{E}=\hbar \nabla \psi_{E} ; \quad p_{S T}{ }^{*}=-\hbar \nabla \ln \left(\psi_{E}\right)
\end{gathered}
$$

The last equations could be related to the Dirac equation, given a momentum $p_{S T}$ connected to the Hamiltonian and the gradient intended over ict in the present elaboration cycle. 
In the absence of momentum in space from the CoM reference frame, in each instant a particle could emerge at a distance $i r=i n L=i L e^{\varphi}$ from its $\mathrm{CoM}$, but most commonly at $i r_{c}$ (Compton radius, with a resulting equivalent wavelength $\lambda_{c}=2 \pi r_{c}$ ). From the relation of the Compton radius and the rest mass with the absolute scales of reference for length $L$ and for mass $M$, we could identify a characteristic dimensionless number, as a proper tempo or characteristic logarithmic scale $\varphi_{c}$ of the particle.

$$
\begin{gathered}
\frac{\lambda_{c}}{2 \pi L}=\frac{r_{c}}{L}=\frac{M}{m_{0}}=n_{c}=e^{\varphi_{c}} \\
|L \nabla \ln (R)|=L \frac{p_{T}}{\hbar}=\frac{p_{T}}{p_{0}}=\frac{m_{0}}{M}=\frac{L}{r_{c}}=\frac{1}{n_{c}}=e^{-\varphi_{c}}
\end{gathered}
$$

From the perspective of the CoM and a support in ict with positive imaginary time, we can describe the amplitude of the wave function of a static massive particle in a given cycle as a Boltzmann distribution. This relation shall be interpreted as an equilibrium between the particle's momentum in time and the global reference of action $\hbar$ and leads to an emergence in the imaginary axis of spatial distance from the CoM with max entropy.

$$
R=\frac{1}{r_{c}} e^{-\frac{r}{r_{c}}}=\left(\frac{p_{T}}{\hbar}\right) e^{-\left(\frac{p_{T}}{\hbar}\right) r}=\frac{1}{L} \frac{p_{T}}{p_{0}} e^{-\left(\frac{p_{T}}{p_{0}}\right) \frac{r}{L}}=\frac{1}{L}\left(\frac{1}{n_{c}} e^{-\frac{n}{n_{c}}}\right)=\frac{1}{L} e^{-\left(\frac{n}{n_{c}}+\varphi_{c}\right)}=\frac{R_{m}}{L}
$$

In each present cycle, the information related to the degree of freedom of the particle in its emergence in space (imaginary distance from the CoM) is encoded in $\boldsymbol{T}_{k}$ as an associated potential "cost" and consequent possible action, in a manifested trembling and uncertainty. In a 3D-space foliation, the probability of emergence of the particle at absolute distance $r$ from its CoM depends on the momentum in time and could be calculated, for $n>0$ as:

$$
\wp\left(\text { ir }=\operatorname{inL} \mid \operatorname{CoM}, \frac{1}{n_{c}}\right)=\frac{1}{2 \pi n_{c}} 4 \pi|i r R|^{2}=\frac{1}{2 n_{c}}\left(\frac{2 r}{r_{c}}\right)^{2} e^{-\frac{2 r}{r_{c}}}=\frac{1}{2 n_{c}}\left(\frac{2 n}{n_{c}}\right)^{2} e^{-\frac{2 n}{n_{c}}}
$$

This distribution assigns a probability to each possible path from $-T$ and $+T$ at absolute distance $r$ from the CoM along ict, in total forward and backward wave and bundle of CTC.

\section{At the root of the matter}

We have identified, in the hypothetic description of a massive particle through the amplitude of its wave function and the momentum in time, a proper tempo $1 / n_{c}$ characteristic of the particle. We have related the proper tempo to the degree of freedom and apparent trembling in the emergence in space from the CoM at each cycle of $\boldsymbol{T}_{\boldsymbol{k}}$.

The proper tempo comes from the confrontation of the particle's momentum in time with the Absolute Time "common beat" (reference of action $\hbar$ ) and drives the probabilistic emergence in ict of the energy-momentum following a Boltzmann distribution.

The description of a particle through the proper tempo (derived from a logarithmic gradient of the wave-function) is intended as an analysis of scale of the particle's info of non-locality in the imaginary space, as better elaborated in the coming chapters.

The momentum in time should be intended as the "root" of the non-local potential of the particle in the imaginary space from an absolute perspective "at infinity" (equivalent to the points at $-T$ and $+T$ on the $\boldsymbol{T}_{k}$ axis from the CoM at $2 k T$ and given $i c t \rightarrow \infty / \mathrm{k}$ as beyond the current maximum imaginary time extension, not a mathematical infinity). It offers a synthesis of the non-local potential considering the quantum uncertainty as a constrained freedom in the emergence, equivalent action and resulting probabilistic observed behavior. In the context of a thick present, the momentum in time could also be interpreted as an absolute view on "what it was" (in terms of persisting info rooted at the boundary between the cycle $k$ and $k-1$ ) and a self-centered but impartial perspective on "what it could be" in the emerging imaginary space (following a weighted freedom in the Boltzmann distribution of the paths from $-T$ to $+T$ crossing the ict axis in a possible point of emergence).

Given the ontological nature of the thick present potential $\boldsymbol{T}_{k}$, the momentum in time is intended as a "root" from - $T$ of the information of "existence" of a particle in the previous cycle, mirrored from $+T$ as a consistent information persisting in the coming cycle. 
Given a symmetrical description in the thick present and considering the proposed connection between non-locality in the imaginary space and CTC, we can as well describe the information related to the non-locality in the emergence of a particle in the imaginary space in each cycle as a probabilistic bundle of CTC and undefined paths from $-T$ to $+T$. The entanglement paths connecting distant quanta of space through CTC are peaked in probability at the ones passing through the imaginary points at absolute distance $r_{c}$ from the CoM in the imaginary space. In this representation, it is the non-local information potential entangling distant quanta of space that defines a particle in the thick present.

The entanglement among quanta of space shall be pictured as quantum tunnels (ER bridge) to the imaginary location where the information of the static mass could manifest as a point-particle. The "point-particle" should be considered as just an event at a given cycle. From a "root in $\boldsymbol{T}_{\boldsymbol{k}}$ ", an event is a point where the potential in $\boldsymbol{T}_{\boldsymbol{k}}$ manifested in ict as a localized momentum in spacetime, collapse of all possible CTC to an actual path from $-T$, causally affecting the evolution as a new fixed point $i c t_{e} \mid k$ mirrored coherently from $+T$.

In Fantasia, a particle eventually seems, in its proper tempo, an elementary vibration of spacetime itself, as a group of imaginary space quanta (or virtual black holes in Hawking's description) entangled on a common proper beat in respect to the global beat of reference. At least in Fantasia, thanks to a description of non-locality $(E R)$ and of entanglement $(E P R)$ through CTC in a thick present, we can extend the famous $E R=E P R$ of holographic theories to a wider $E R=E P R=C T C @ \boldsymbol{T}_{k}$, describing a logically consistent information encoding the freedom of the present potential, towards a truly "unbroken wholeness of the entire universe" with no strings attached or deterministic hidden variables.

In Fantasia, matter is a gradient of information related to the local potential in a volume of imaginary space entangled on a common momentum in time. It might be worth to note that the momentum in time, as a root from - $T$ of the persisting information potential, seems always well-defined at the CoM in the thick present, independently from any event at a specific location in ict in the current cycle, leading to a well-defined metric from the CoM. If entanglement in space is the choice among paths from $\pm T$ across distant quanta and, as matter, curves the otherwise flat ict in a non-local potential summarized at the CoM, then we should probably stop looking for superposed metrics in non-locality and spatial entanglement only, and focus more on undefined causalities of massive objects, to create distributed imaginary CTC and entanglement in both space and time, and possibly more explicit quantum phenomena.

\section{A hologram from a slice of time}

The following figures illustrate the introduced concepts in a pictorial representation, highlighting the emergence in $i c t=i L e^{\varphi}$, the probability density from the CoM in log-scale derived by the momentum in time, and the corresponding most probable CTC, in a simplified 1D-section of the space foliation.

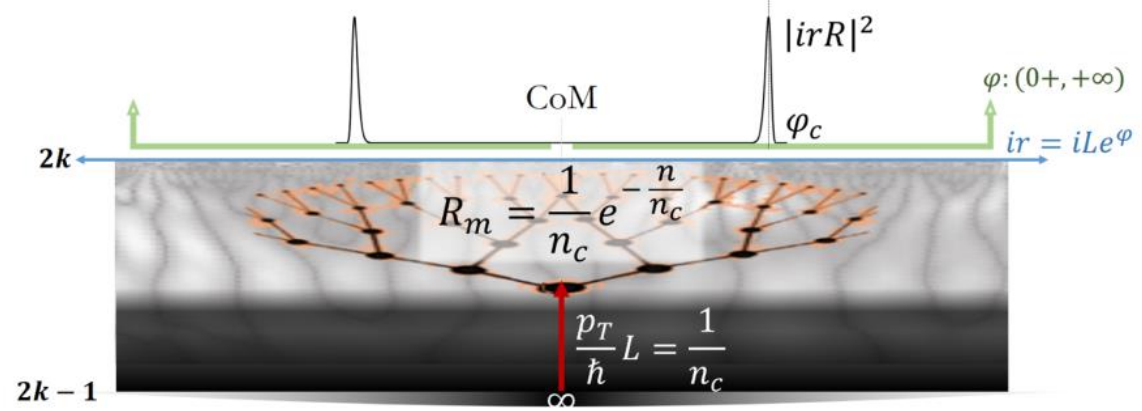

Figure 4. Pictorial representation of the emergence of a particle in each cycle at absolute distance $r$ $\left(i L e^{\varphi}\right)$ from its CoM along the ict axis (density as $|i r R|^{2}$ on a log-scale through the variable phase $\varphi$ ). The momentum in time is interpreted as a root from the perspective at $-T$ in $T_{k}$ (equivalent to infinity in the spatial dimension) of the potential related to the non-locality in the emergence in ict. Considering a logarithmic perspective on the emergence in ict, a particle could be intended as a vibration mode at a given scale of the spacetime fabric. 


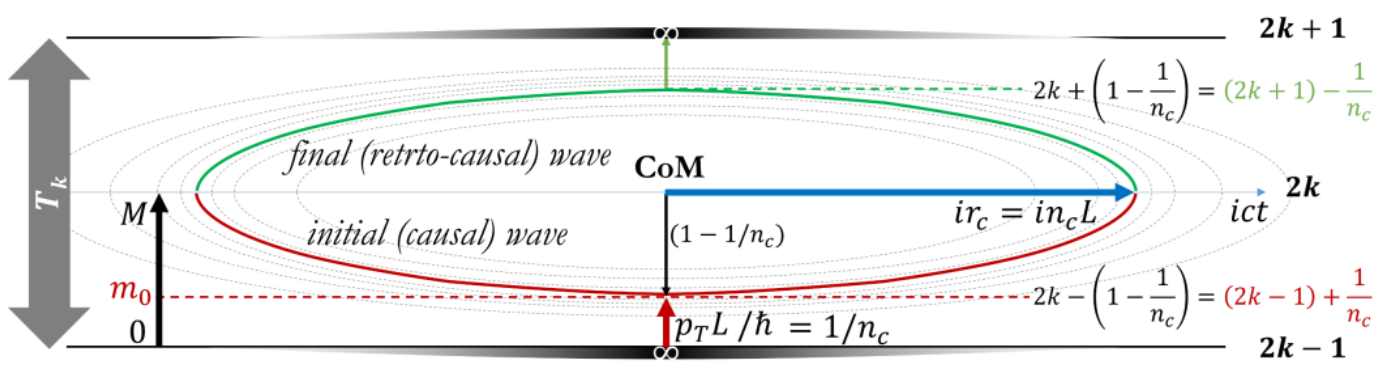

Figure 5. Schematic representation of the information of a particle as momentum in time and bundle of CTC (paths connecting $-T$ and $+T$ ). The CTC of max probability crosses the points in ict at absolute distance $r_{c}$ from the CoM. The momentum in time is represented as vectors from the boundaries of the present at $2 k-1$ and $2 k+1$ (equivalent to a view from infinity in space).

The proposal outlined so far is intended as a draft framework for the interpretation of spacetime and of the matter that curves its geometry through information, described as the entanglement among quanta of space, separated by an imaginary time of motion and evolving synched on a common beat in a global cycle. After having dared much, in the next chapter we will elaborate more on the possible interpretations that the proposed toy model could offer. Still far from a mathematical derivation, it is worth to consider several different representations of the proposed ideas, as multiple perspectives able to extrapolate, from a simple and draft framework, additional insights.

\section{Perspectives on Fantasia}

\section{A packet in a global network}

In an ICT description of the entanglement among the quanta of space, Fantasia spacetime could be considered as emerging from an interconnected network acting as a quantum computational memory (made of CTC or memory-loops) that encodes and projects the full space of possibilities on a globally synched cycle. An elementary massive particle could be described as a network of additional non-local correlations in the imaginary space within the global information. It is a local network in a greater Network, persisting as a smaller packet of coherently entangled imaginary "Planck oscillators".

Within Fantasia, the ensemble of the entangled links related to the elementary particle identifies a volume of imaginary space on a "common beat", like a local "persisting tune". From an ICT perspective, the momentum in time could then be interpreted as well as a "self-decoding reference" relative to the global reference of action in the present cycle.

In a wavelet decomposition of the potential in a local volume of space, it could be described as the local ground level of summarized information "at the heart" of the CoM, representing the information common to all the imaginary quanta in the global network entangled in the same elementary particle, as well as the scale of the resonating elementary wavelet.

\section{Self-similarities and invariances}

In an ICT description of Fantasia, the Absolute Time $T_{k}$ could be intended as a quantum memory axis that elaborates the information of spatial correlation emerging in ict as if the quanta of space were connected in a single global network. In this sense, the encoding of the information in $T_{k}$ can be seen as a "holographic compression algorithm" for all the complexities and degrees of freedom in the states emerging on the boundary ict. Looking for a synthetic perspective, a wavelet analysis could exploit the spatial scale invariances and fractal self-similarities of natural phenomena (which usually follow a lognormal distribution, as highlighted by Kolmogorov in [23] and others in [24]), allowing the most efficient representation of the potential freedom in the imaginary axis of distance. The logarithm is also the function defining the information content and a log-perspective seems better suited for natural information elaboration. 
The Absolute Time, in its inversion of perspective in the synthesis of the information of mass in the momentum in time, has a log-perspective on the entanglement in space. Scanning in each present cycle all spacetime imaginary distance scalar modes along $1 / n$ (going from $2(k-1) T$ and $2(k+1) T$ to $2 k T$ in an atomic evolution) seems equivalent to an integral spanning from infinity in the space of $n$ and eventually a log-perspective on $i c n T$. We should then describe the analysis of scale of the vibrating modes in the emerging fabric as a wavelet analysis performed in the bulk regions and derived as an integral over the inverse of the ict distance (with shorter and local modes closer to the boundary ict@2kT, while longer ones accounted deeper in the dual symmetric bulks of $\boldsymbol{T}_{k}$, up to $\infty$ mapped at $\pm T$ from $2 k T$ ). On the boundary ict $@ 2 k T$, from any imaginary quanta of space as a possible CoM of localized information, we can "close the paths" in opposite directions in the imaginary time of motion with a unique point at infinity. This closure at infinity can be interpreted, from a global perspective on ict, as a shift invariant description of space that sets the origin and infinity as any opposite points on a closed path of imaginary distance.

The combination of a wavelet analysis of the information of scale in ict (exploiting the scale invariance of natural phenomena) and of a shift invariant localized description along the imaginary space is equivalent to a Dual-Tree Complex Wavelet Transform (DTCWT), mathematically described in Ref. [25]. A DTCWT, in the encoding of the entanglement among virtual quanta of space separated by an imaginary time of motion, exploits both scale and phase shift invariance and optimally compresses the imaginary empty spaces.

For an efficient synthesis of the information of entanglement among quanta of space in a full $i$ ct axis, a DTCWT with an ideal filter bank transfer function $1 / t$ (acting as the real memory filter at different scales of $i c t$ ) and the corresponding Hilbert transform pair $\delta(t)$ (scanning the phase variations in the imaginary time of motion) is then proposed as the "encoding algorithm" of Fantasia spacetime in the symmetric bulks of $\boldsymbol{T}_{k}$.

\section{Stability, consistency and existence}

Following a holographic perspective, we have pictured the ict dimension (starting from a CoM at $2 k T$ ) as on the boundary between the bulks of $\boldsymbol{T}_{k}$. We have also mapped the points at $(\infty \mid k)$ in ict to the perspective at $-T$ and $+T$ in the symmetric bulk regions of $\boldsymbol{T}_{k}$, as a far distant origin of the information emerging as space from a global perspective.

In the proposed model, the entanglement of space associated to an elementary particle is mathematically expressed in the 2 symmetric bulks as the inverse of the information of distance along the emerging imaginary space, as a spatial frequency and "common beat" related to the momentum in time in respect to the global reference of action.

The inversion of perspective in $1 / n$ in a time symmetric description from $-T$ and $+T$ suggests interpreting $\boldsymbol{T}_{k}$ as a pair of Anti-de-Sitter (AdS) symmetric bulk regions encoding the information of entanglement and curvature in ict. It would be interesting to mathematically verify if the surface at the boundary between the 2 symmetric AdS bulk regions could be considered flat and stable at large scales in ict.

Beyond flatness at large scales, to allow a consistent evolution and an emerging causality, the information potential in $T_{k}$ shall not be under-determined (undecidable) or over-determined (contradictory), as required in the hypothesis of logical consistency. Logical consistency is considered assured thanks to the combination of a forward and a backward-evolving contribution, existing as a potential in the two hemicycles of a CTC. The encoding of the non-local potential of matter through CTC seems suggesting a deeper relation between the logical consistency of the information and the resulting curvature in the imaginary space. Given that in ICT loops are actually the most basic circuits for memory allocation, these CTC could be seen as "memory loops" that connect different quanta in the emerging space, assuring the logical consistency of the non-local information with a consistency-check forward and backward in time within the thick present cycle. Note that in each quantum of space in ict, these memory loops cross perpendicularly the spatial axis of the imaginary distances, as the usual space and time orthogonality in a conformal description that preserves angles.

Figure 6 illustrates the description of the thick present introduced in a pictorial form. 


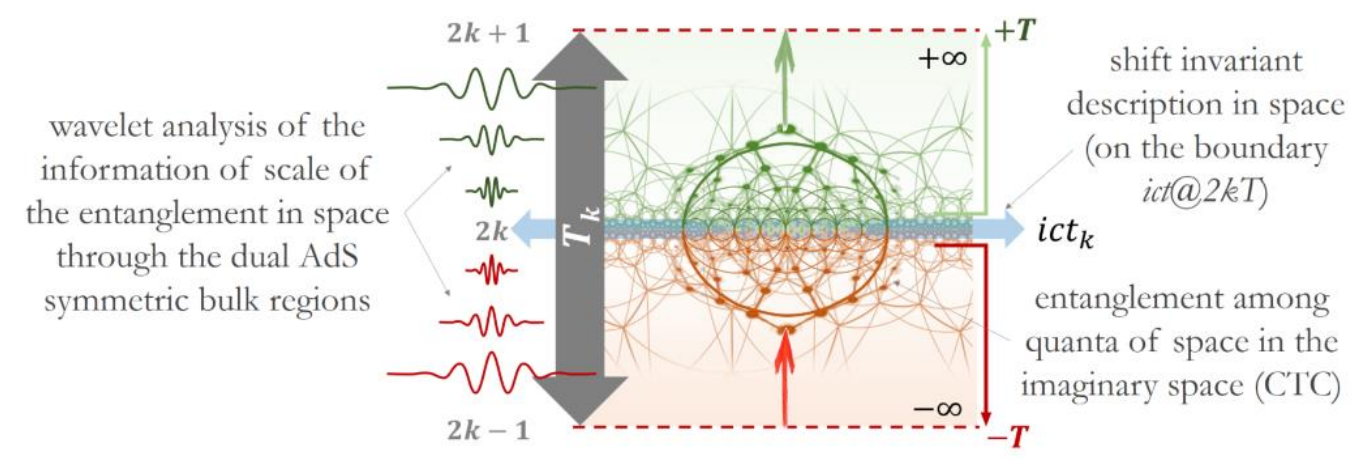

Figure 6. 1D-spatial section of the thick present. When no matter is present, distance is pictured as an imaginary and shift invariant time of motion in a flat ict. Massive particles are described as distant quanta of space entangled through a bundle of CTC on a "common beat" (momentum in time) synthetized as an equivalent information of scale in $\boldsymbol{T}_{k}$. Different scales of CTC are represented, highlighting the log-perspective of $\boldsymbol{T}_{k}$ on the information of non-local correlations, as in a DTCWT. The entanglement among quanta of space is eventually encoded in $\boldsymbol{T}_{k}$ as in a pair of AdS symmetric bulk regions, rooted at $-T$ and $+T$ and extending to the common boundary ict@2kT

Matter information in Fantasia has been related to a vibration mode of the spacetime fabric, which persists as a probabilistic bundle of CTC (representing the forward and backward contribution in the present) that entangle distant quanta of space in a common momentum in time. This information is manifested in the emergence from the CoM as a random degree of freedom shaped as a Boltzmann distribution and persists along the evolution cycles as a potential in a memory axis $\boldsymbol{T}_{k}$ that eventually becomes ontological. Considering the ontological nature of the information in the Absolute Time, the derived gravitational information could metaphorically be interpreted as the "proof of existence" in the previous cycle of a static mass, rooted at $-T$ as a forward wave and consistently mirrored from $+T$ as a backward wave in a logically consistent persisting information.

In the proposed model, gravity is always attractive as the information of distance in ict is always encoded in $T_{k}$ with an absolute perspective. The gradient of entanglement information should be interpreted as a slope towards regions showing a "slower beat", proof of additional information in an absolute sense.

\section{Symmetries in Fantasia}

\section{Spin to explore ict}

From the CoM perspective, space extends radially in all directions on an imaginary time of motion coherent with the speed of light, defined as the ict axis, with $t=n T>0$ (considering the case $n=0$ as the CoM itself). The symmetry in ict along the 2 opposite sides in a given linear direction from the CoM perspective could be related to the possible opposite values of a quantum spin measurement in the normal direction basis $|\uparrow\rangle$ and $|\downarrow\rangle$. In the context of an evolution along thick present instants, the spin $\hbar / 2$ of fermions could be related to a $2 * 2 T$ revolution needed, in respect to the $2 T$ or $2 \pi$ thickness of the present, to explore the full space of possibilities along $i c t$ with both perspectives.

In a global symmetry in $i c t_{|\uparrow \downarrow\rangle}$ emerging from the CoM, the CoM reference frame itself could be defined following the momentum in time as a root and an imaginary path from the perspective at infinity in space.

Metaphorically, considering a particle as a vibration in an interconnected and entangled spacetime fabric, we could say that the particle identifies its CoM in the emerging universe though its image projected "along threads from infinity" (as in Celestial Spheres).

This metaphor could be easily related to the "belt metaphor". With a "belt" anchored on a book-particle at one end and "at infinity" in our hand on the other side, we will need a $4 \pi$ rotation for a full revolution, as for a spin $1 / 2$ cycle in comparison to the thick present cycle. Figure 7 illustrates the metaphor introduced in a pictorial form. 


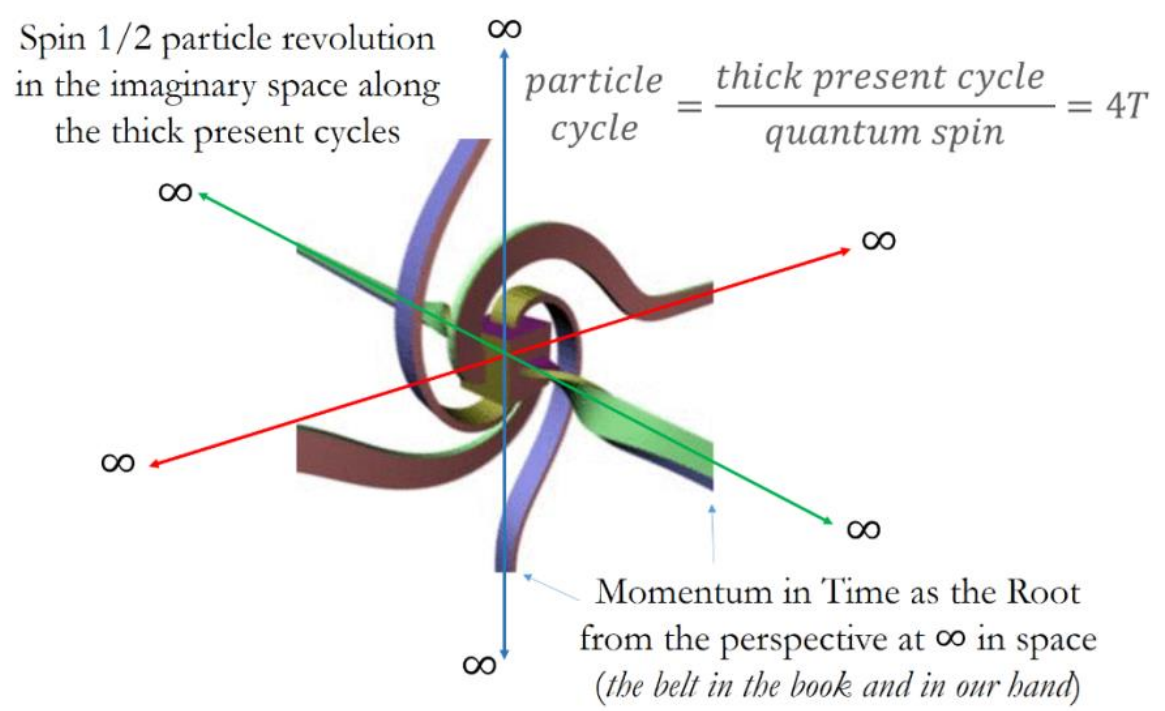

Figure 7. Pictorial representation of a particle CoM revolution cycle of $4 \pi$. The momentum in time is pictured as roots/belts from infinity of the information of scale of the particle. These belts are symmetric along the degrees of freedom spanning the $4 \pi$ perspective from the CoM. Animated version available at en.wikipedia.org/wiki/Orientation_entanglement\#/media/File:Anti-Twister.gif

\section{Anti-matter in $\boldsymbol{T}_{k}$}

In a time-symmetric description within the thick present, we can identify a central imaginary boundary ict and 2 symmetric bulk regions extending from ict to the opposite extremes at $-T$ and $+T$, which close the thick present.

A massive particle's information could be decomposed in a symmetric fashion in the 2 bulk regions, as a forward and backward evolving wave representing the 2 hemicycles of the CTC bundle crossing perpendicularly the boundary ict@2kT.

Causality progresses from past to future cycles, as events accumulate in the past and space expands in its imaginary dimension in the future, with a consequent gradient in entropy (increase in the minimum information needed to describe the present potential) in a resulting thermally oriented emerging time. We can identify then a causal propagation, from - $T$ in $\boldsymbol{T}_{k}$ to the boundary at ict, and a retro-causal one from $+T$ "back to" ict@ $2 k T$.

We could relate this symmetry in $T_{k}$, broken by the gradient of entropy, to the difference between matter and anti-matter.

From this perspective, we could consider antimatter as opposite in the Absolute Time-matter, phasing with inverted hemicycles along the evolution cycles. It could be interpreted as if the initial and the final waves occurred in opposite direction in respect to the arrow of causality and the momentum in time had consequently "opposite sign" in respect to matter (as illustrated in Figure 8).

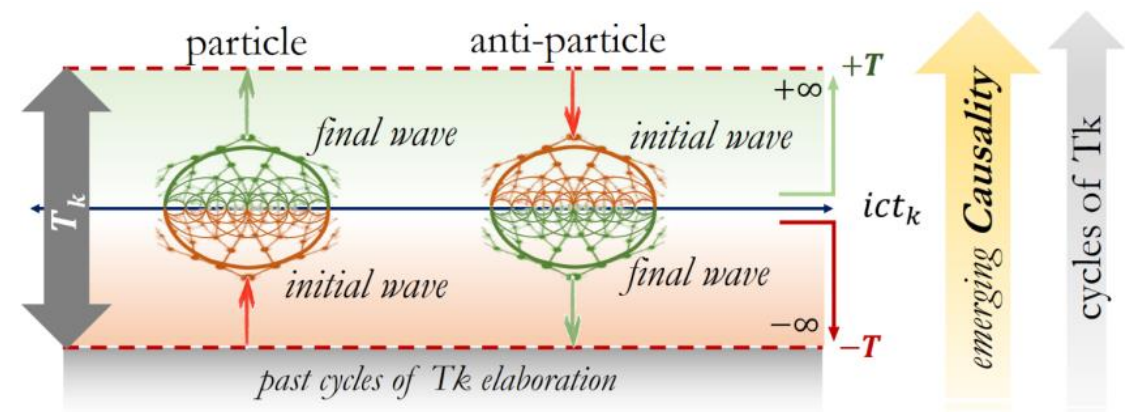

Figure 8. Schematic representation of an elementary particle and its corresponding anti-particle with "inverted momentum in time". The wave function evolves on opposite hemicycles in the thick present, as if propagating "backwards in time" 
We could metaphorically say that antimatter is not in an underlying "Dirac sea" but comes from the reference at $+T$ in respect to the arrow of causality in ict @ $2 k T$, as if dropping like a "Dirac rain" from the future and as if evolving backwards in time in the thick present. This perspective gives a possible physical interpretation to the Feynman-Stueckelberg proposal, which considers anti-particles as matter evolving "backwards in time".

The thermodynamic arrow of time, emerging along causality and entropy gradients from past to future cycles, could be related to a minimal deviation from a flat ict at infinity.

The "anti-causal" nature of antimatter might be connected to a possible greater instability (as a pendulum oscillating on an unstable equilibrium point), potentially explaining the slight asymmetry of antimatter abundance in respect to normal matter, in Fantasia as in any other similar Universe.

\section{Synthesis and outlook}

In the context of a spacetime described through information, we have proposed a toy model of the universe, named Fantasia.

For a physically implementable solution, we have required the information to be finite, discrete, relative to universal scales of reference and logically consistent at a global level. Following the insights from a previous work, we have considered evolution in atomic thick present instants and modeled spatial distance through an imaginary time of motion between distant quanta of space (virtual Planck-mass oscillators), in an emerging ict axis representing a degree of freedom from any possible CoM.

Fantasia spacetime emerges in each thick present instant (of duration $2 \mathrm{~T}$ every $2 k T$ ) as a flat spacelike foliation along $i c t$, and it is curved by the additional entanglement in space.

In its cyclic evolution every $2 k T$, the thick present identifies a universal reference for a quantum of action and represents the "fastest event" to evaluate differences (equivalent to a spacetime information "sampling rate").

We have described the entanglement among the quanta of space through CTC developing in the time symmetric present, and as an information potential related to an open choice, expressed as non-locality in the foliation and encoded in the thickness of each instant.

The thickness of the present has been pictured as a symmetric "cyclic memory" axis named $T_{k}$ or Absolute Time, orthogonal to the imaginary space axis and encoding the information of entanglement in each instant. It is symmetric as it extends from $(2 k-1) T$ to $(2 k+1) T$ in respect to the emergence of ict@2kT, it is a "cyclic memory" given the thick present as a cyclic elaboration of the information potential encoded in $T_{k}$ (as in a "quantum memory"), and it is "absolute" as it is related to absolute references (characteristic of the universe), like the Planck units and the global quantum of action identified by the thick present cycle. In a holographic perspective, we have described $i c t @ 2 k T$ as the boundary between the 2 symmetric bulk regions identified in $T_{k}$ as $(2 k-1) T-2 k T$ and $(2 k+1) T-2 k T$ respectively.

After having defined the high-level framework, we have conjectured how the information potential of massive particles could be represented.

We have proposed a description of particles as quanta of space entangled on a common "proper tempo". This proper tempo has been derived through a logarithmic gradient of the amplitude of the wave function and has been described as a "momentum in time" in respect to the global reference of action, connected to the mass of the particle.

The momentum in time defines the shape of a Boltzmann distribution (connected to an emergence in the imaginary space with maximum entropy) and has been interpreted as an "analysis of scale" of the information of non-locality of the particle, encoded in $\boldsymbol{T}_{\boldsymbol{k}}$ from the perspective at infinity in space (equivalent to $\pm T$ in $T_{k}$ ). In this context, a particle emerges on the surface of a sphere of which the imaginary radius is encoded as a momentum and equivalent curvature in the orthogonal atomic cyclic memory axis of the Absolute Time. Given a causality orientation and the ontological nature of $\boldsymbol{T}_{k}$, the momentum in time has been described as a "root" in $T_{k}$ from $-T$ of the information of "existence" of a particle in the previous cycle of Fantasia, mirrored from $+T$ for a persisting info in the coming cycle. 
In the last part of this paper, we have further elaborated on the possible interpretations of the potential encoded in the thick present and on the symmetries in Fantasia.

In a connected universe, we have first considered particles as packets in a global network. Then, the description of the potential through a log-perspective on the information of scale in space has led us to consider, as an efficient encoding of such info, a wavelet analysis. This encoding is elaborated in the symmetric bulk regions as a filter $1 / t$ on the imaginary distances in ict and could be the most efficient synthesis for natural phenomena representation (often showing fractal self-similarities). Considering a shift invariant description in the imaginary space, the resulting encoding of the information in $T_{k}$ has been intended as a DTCWT, with $1 / t$ and $\delta(t)$ as Hilbert transform filters pair.

Following the math of the proposed encoding in $T_{k}$, we have then described the 2 regions identified in the intervals ( $2 k-1) T-2 k T$ and $(2 k+1) T-2 k T$ in $\boldsymbol{T}_{k}$ as 2 symmetric AdS bulks, sharing a boundary in $i c t @ 2 k T$ that may result flat and stable at infinity (to be verified).

Beyond stability, also logical consistency and its connection with entanglement and the information of matter through CTC in a time symmetric description seemed to play a crucial role in Fantasia. These considerations led us to describe the information of gravity as the "proof of existence" of the particle, connected to the coherent, stable, and consistent information encoded in the ontological Absolute Time cyclic memory axis.

Finally, we have focused on the additional emerging features derived from the basic symmetries in Fantasia.

We have connected the quantum spin of a particle to its revolution in the imaginary space in respect to the global cycle of the thick present (identifying in the symmetries in ict the possible outcomes of a spin measurement), and conjectured a connection between matter/anti-matter and the symmetry in $\boldsymbol{T}_{\boldsymbol{k}}$.

Matter particles have been considered as evolving in line with the gradient in entropy and causality orientation, while antimatter has been pictured with the initial and final waves oriented in the opposite direction in $T_{k}$ (as a pendulum in an instable equilibrium given the causal gradient), leading to a possible greater abundance of matter in respect to anti-matter.

Fantasia has been proposed as a simple holographic model that might give insights on how our Universe, when described through the lens of information, could be pictured. Starting from simple assumptions for a physical implementable solution has helped to define some of the primitive emerging features of spacetime, such as an extended axis of a perceived causal and relativistic time, an evolving imaginary coherent space of distances and possibilities, and matter particles from the additional entanglement of such space.

Even if several mathematical derivations are missing, the simple model introduced seems to offer a promising path for a better understanding on the nature of spacetime as well as a consistent new interpretation for several concepts in QM foundations.

Beside an explicit connection with GR, a full description of bosons' fields in the geometry proposed will help completing the picture for a consistent model of our Universe.

Beyond QM foundations and a quantum theory of time, the author is interested in a more profound comprehension of the most basic principles that govern our Universe to identify more efficient ways of producing energy.

Currently, fusion may be the most promising path. Hopefully, a deeper understanding of the nature of spacetime and the behavior of particles through information will offer new efficient ways to overcome the global energy production and distribution problem.

In a world running towards ecological disasters, with always less chances to prevent an excessive warming, this kind of goal should eventually promote a renewed and urgent effort of the global scientific community, as well as of the whole human society.

Funding: This research received no external funding.

Conflicts of Interest: The author declares no conflict of interest. 


\section{Abbreviations}

The following abbreviations are used in the contribution:

CoM Center of Mass

CTC Closed Time-like Curves. Closed paths in spacetime. No events occur on their path and time has no causal or thermodynamic orientation along them. They represent an information potential in the superposition of the outcomes of an open choice (eg: direction of travel along the curve). When developing between $-T$ and $+T$ in the thick present, they cross the imaginary time axis in distant quanta of space that result connected (non-locality, entanglement in space). When developing in the imaginary space foliation they represent indefinite orders of traversed quanta of space (undefined causality, entanglement in time). They are considered in the paper as "memory loops"

EPR Einstein - Podolsky - Rosen. Reference to the 1935 famous contribution on the incompleteness of QM. Intended in the paper as EPR paradox, a synonym of entanglement and entangled particles (EPR pairs)

ER Einstein - Rosen bridge. Quantum wormhole connecting far regions of spacetime. Intended in the paper as entanglement between distant quanta in the imaginary space foliation (non-local information potential)

GR General Relativity (theory of)

ICT Information and Communication Technologies

ict Imaginary coherent time. Described as the imaginary time of motion (it) at the speed of light $(c)$ between distant quanta of space. It is proposed in the paper as an imaginary axis of spatial distance and, in absence of additional information potential, it defines a flat space foliation emerging in the present

OPT Operational Probabilistic Theories. Description of QM from first principles based on Information Theory

PI Path Integral formulation of QM

QG Quantum Gravity

QIS Quantum Information Science

QM Quantum Mechanics

$T_{k} \quad$ Absolute Time memory. Information potential existing in the thick present (at current evolution cycle, after $2 k T$ instants from the origin of the Universe). Time symmetric description from $(2 k-1) T$ and $(2 k+1) T$ of entanglement in space (non-local potential in the imaginary space foliation emerging at $2 k T$ ) and of entanglement in time (undefined causality and indefinite time orders)

TSVF Two State Vector Formalism. Time-symmetric description of QM

UCO Undefined Causal Orders. Entanglement in time (order) 


\section{References}

[1] A. Capurso, "The potential of a thick present through undefined causality and non-locality," Entropy, vol. 24, no. 3, pp. 410 - doi.org/10.3390/e24030410, 2022.

[2] N. Gisin, "Indeterminism in Physics, Classical Chaos and Bohmian Mechanics. Are Real Numbers Really Real?," Erkenn, 2019.

[3] N. Gisin, "Classical and intuitionistic mathematical languages shape our understanding of time in physics," Nature Physics, vol. 16, pp. 114-116, 2020.

[4] C. Mariani and G. Torrengo, "The Indeterminate Present and the Open Future," Synthese, vol. 199, pp. 3923-3944 - doi.org/10.1007/s11229-020-02963-y, 2021.

[5] A. Schlatter, "On the Principle of Synchronization," Entropy, vol. 20, pp. 741 doi:10.3390/e20100741, 2018.

[6] A. Schlatter, "On the Reality of Quantum Collapse and the Emergence of Space-Time," Entropy, no. 21, pp. 323 - doi:10.3390/e21030323, 2019.

[7] A. Schlatter, "On the Foundation of Space and Time by Quantum-Events," Found Phys, vol. 52, pp. 7 - https://doi.org/10.1007/s10701-021-00526-w, 2022.

[8] L. Smolin and C. Verde, "The quantum mechanics of the present," arXiv:2104.09945v1, 2021.

[9] V. Vedral and C. Marletto, "Gravitationally-induced entanglement between two massive particles," Phys. Rev. Lett., vol. 119, no. 24, 2017.

[10] C. Rovelli and M. Christodoulou, "On the possibility of experimental detection of the discreteness of time," Front.in Phys., vol. 8, 2018.

[11] Ä. Baumeler, "Causal Loops: Logically Consistent Correlations, Time Travel, and Computation," PhD Thesis, Università della Svizzera Italiana, 2017.

[12] A. Baumeler, A. S. Gilani and J. Rashid, "Unlimited non-causal correlations and their relation to non-locality," arXiv:2104.06234v1, 2021.

[13] P. Blasiak, E. M. Pothos, J. M. Yearsley, C. Gallus and E. Borsuk, "Violations of locality and free choice are equivalent resources in Bell experiments," PNAS, p. e2020569118, 2021.

[14] J. Conway and S. Kochen, "The Free Will Theorem," Foundations of Physics, vol. 36, p. 14411473, 2006.

[15] C. F. Paganini, "No events on closed causal curves," arXiv:2005.05748v4, 2021.

[16] L. M. Procopio, A. Moqanaki, M. Araújo, F. Costa, A. Calafell, E. G. Dowd, D. R. Hamel, L. A. Rozema, Č. Brukner and P. Walther, "Experimental superposition of orders of quantum gates," Nature Communication, vol. 6, p. 7913, 2015.

[17] G. Rubino, L. A. Rozema, F. Massa, M. Araújo, M. Zych, Č. Brukner and P. Walther, "Experimental Entanglement of Temporal Orders," Quantum Information and Measurement, vol. Quantum Technologies, p. paper S3B.3, 2019.

[18] M. Zych, F. Costa, I. Pikovski and C. Brukner, "Bell's theorem for temporal order," Nature Communication, vol. 10, no. 3772, 2019.

[19] G. Chiribella, "Indefinite causal order enables perfect quantum communication with zero capacity channels," New J. Phys., vol. 23, no. 033039, 2021.

[20] S. W. Hawking, "Quantum gravity and path integrals," Phys. Rev. D, vol. 18, no. 6, pp. 17471753 - DOI:10.1103/PhysRevD.18.1747, 1978.

[21] S. Esposito, “On the role of Spin in Quantum Mechanics," Found Phys Lett, vol. 12, p. 165-177, 1999.

[22] G. Salesi, "Spin and Madelung fluid," Modern Physics Letters A, vol. 11, no. 22, 1996.

[23] A. N. Kolmogorov, "On the logarithmic normal distribution law of particles with dimensions of fragmentation," Dok. Acad. Nauk SSSR, vol. 31, p. 99, 1941.

[24] E. Limpert, W. A. Stahel and M. Abbt, “Log-Normal distributions across the Sciences: Keys and Clues," BioScience, vol. 51, no. 5, pp. 341-352, 2001.

[25] I. Selesnick, R. Baraniuk and N. Kingsbury, "The Dual-Tree Complex Wavelet Transform," IEEE SIGNAL PROCESSING MAGAZINE, Vols. 1053-5888/05, 2005. 